\title{
Article \\ Analyzing the Effects of Parameters for Tremor Modulation via Phase-Locked Electrical Stimulation on a Peripheral Nerve
}

\author{
Jeonghee Kim ${ }^{1,2,3, *}$, Thomas Wichmann ${ }^{4,5}$, Omer T. Inan ${ }^{6}\left(\mathbb{D}\right.$ and Stephen P. DeWeerth ${ }^{6,7}$ \\ 1 Department of Engineering Technology and Industrial Distribution, Texas A\&M University, \\ College Station, TX 77843, USA \\ 2 Department of Multidisciplinary Engineering, Texas A\&M University, College Station, TX 77843, USA \\ 3 Department of Electrical and Computer Engineering, Texas A\&M University, College Station, TX 77843, USA \\ 4 Department of Neurology, Emory University, Atlanta, GA 30322, USA; twichma@emory.edu \\ 5 Neuropharmacology and Neurologic Diseases, Yerkes National Primate Research Center at Emory University, \\ Atlanta, GA 30329, USA \\ 6 School of Electrical and Computer Engineering, Georgia Institute of Technology, Atlanta, GA 30332, USA; \\ omer.inan@ece.gatech.edu (O.T.I.); steve.deweerth@lehigh.edu (S.P.D.) \\ 7 Department of Biomedical Engineering, Lehigh University, Bethlehem, PA 18015, USA \\ * Correspondence: jeonghee.kim@tamu.edu
}

check for updates

Citation: Kim, J.; Wichmann, T.; Inan, O.T.; DeWeerth, S.P. Analyzing the Effects of Parameters for Tremor Modulation via Phase-Locked Electrical Stimulation on a Peripheral Nerve. J. Pers. Med. 2022, 12, 76 https://doi.org/10.3390/ jpm12010076

Academic Editors: Dag Nyholm and Filip Bergquist

Received: 19 November 2021 Accepted: 31 December 2021

Published: 8 January 2022

Publisher's Note: MDPI stays neutral with regard to jurisdictional claims in published maps and institutional affiliations.

Copyright: (c) 2022 by the authors. Licensee MDPI, Basel, Switzerland. This article is an open access article distributed under the terms and conditions of the Creative Commons Attribution (CC BY) license (https:/ / creativecommons.org/licenses/by/ $4.0 /)$.

\begin{abstract}
Background: Non-invasive neuromodulation is a promising alternative to medication or deep-brain stimulation treatment for Parkinson's Disease or essential tremor. In previous work, we developed and tested a wearable system that modulates tremor via the non-invasive, electrical stimulation of peripheral nerves. In this article, we examine the proper range and the effects of various stimulation parameters for phase-locked stimulation. (2) Methods: We recruited nine participants with essential tremor. The subjects performed a bean-transfer task that mimics an eating activity to elicit kinetic tremor while using the wearable stimulation system. We examined the effects of stimulation with a fixed duty cycle, at different stimulation amplitudes and frequencies. The epochs of stimulation were locked to one of four phase positions of ongoing tremor, as measured with an accelerometer. We analyzed stimulation-evoked changes of the frequency and amplitude of tremor. (3) Results: We found that the higher tremor amplitude group experienced a higher rate of tremor power reduction (up to 65\%) with a higher amplitude of stimulation when the stimulation was applied at the \pm peak of tremor phase. (4) Conclusions: The stimulation parameter can be adjusted to optimize tremor reduction, and this study lays the foundation for future large-scale parameter optimization experiments for personalized peripheral nerve stimulation.
\end{abstract}

Keywords: essential tremor; peripheral nerve stimulation; neuromodulation; parameter optimization; tremor quantification; wearable non-invasive stimulation

\section{Introduction}

Tremor in neurological disorders such as essential tremor (ET) and Parkinson's disease (PD) [1-4] can significantly reduce quality of life for patients [5-7]. This is particularly true for ET, a chronic and progressive neurological disorder. ET typically occurs with movement, with tremor frequencies of $4-12 \mathrm{~Hz}$ [8-12]. This disorder affects about ten million people in the United States. Although tremor movements can be observed in all parts of the body, such as the hands, the head, the voice, the legs, and the trunk, $90 \%$ of ET patients experience arm tremor. Symptoms of ET most commonly appear in people in their 40s and older. Nine percent of people over the age of 60 experience tremor [13,14].

Once patients are diagnosed with ET, the general treatment of their symptoms is pharmacotherapy. Common medications for the disorder are primidone and beta receptor blockers (e.g., propranolol) [15-19]. These medications often help, but $40 \%$ of patients do not derive meaningful benefits, and some experience significant side effects (e.g., fatigue, 
nausea, dizziness, sedation) [18,19]. Another possible therapy is the intramuscular injection of botulinum toxin, specifically in tremors with a dystonic component [20-22]. Tremor patients also have the option of undergoing deep brain stimulation (DBS) therapy [23-29] in which a series of electrodes are implanted into the ventral intermediate nucleus of the thalamus. While effective in most treated patients, the cost of this treatment is high, and the prospect of brain surgery is unattractive for many patients.

Tremor can also be suppressed by functional electrical stimulation (FES), which aims to stimulate arm muscles of the upper limbs [30-36]. FES attenuates tremor in patients with ET or PD. FES at motor threshold, evoking contractions of flexor and extensor muscles either out-of-phase or in phase (co-contraction), modulates tremor. Yet another approach entails the stimulation of afferent pathways [36-48]. In this case, sub-threshold electrical stimulation of afferent pathways is used [37-47]. It is possible that this type of stimulation modulates neurological pathways that produce tremor. Among these studies [48-50], a few (including ours [47]) entailed the use of a wearable setup that applies electrical stimulation to afferent pathways while collecting tremor movements via a motion sensor. One wearable device is commercially available [51] and has been used successfully. The developers of the device did not use real-time parameter adjustment strategies to maximize tremor suppression.

In a previous publication [47], we showed that external electrical stimulation of the radial nerve, administered via a small wearable device, can significantly reduce tremor in ET participants. The prior study analyzed a limited number of combinations of electrical stimulation parameters in an open-loop configuration. As the first step towards development of a closed-loop (self-adjusting) wearable tremor modulation system, we contrasted several varying parameter combinations (differing in amplitude, frequency, and phase alignment to ongoing tremor) in the current study. The overall concept of the closed-loop stimulation parameter optimization for the tremor modulation via peripheral nerve electrical stimulation is shown in Figure 1. Steps 1-4 of the Figure 1 show the overall experimental procedure of this study.

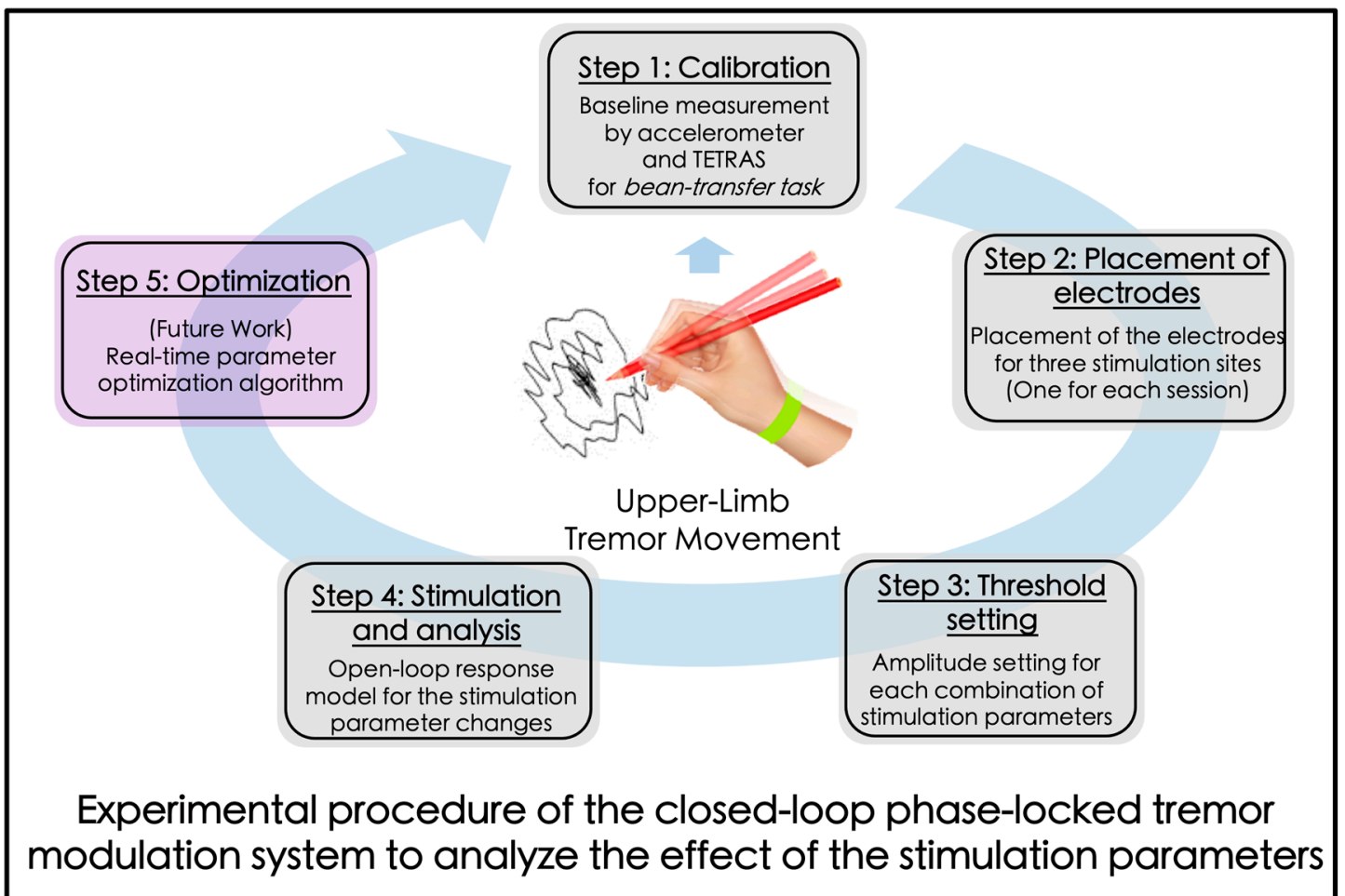

Figure 1. Experimental procedure for the analysis of the effects of the stimulation parameters using the tremor monitor and modulation system. 


\section{Materials and Methods}

\subsection{Experimental Design}

The overall experimental procedure is depicted in Figure 1. We began by collecting the baseline movement to analyze the dominant frequency and the peak amplitude of the subjects' tremor when they performed a "bean-transfer" task, which involved transferring a small object (a medium-sized lima bean) from one plate to another using a spoon (Figure 2). The performance of the calibration procedure was used for the "control (no stimulation)" condition, as well as for the tremor detection algorithm. The bean-transfer task mimicked utensil movements during eating which is one of the most common and important daily activities. The subjects were asked to transfer four beans during a ten-second calibration period. Then, we placed a pair of electrodes on a branch of the designated nerve near the wrist, as shown in Figure 2. To confirm stimulation of the desired nerve branch, we asked subjects to delineate the location of stimulation-induced sensory changes. The stimulation was said to be in good position if it affected sensation in the area highlighted in Figure $3 \mathrm{~b}$.

Once the electrodes were properly placed, we administered electrical stimulation with two combinations of parameter settings (100 or $200 \mathrm{~Hz}$ frequency with $12.5 \%$ of duty cycle) to determine the minimum and maximum amplitudes of the stimulation (threshold setting). The minimum amplitude (low amp) was defined as the sensory threshold (1T), and the maximum amplitude (high amp) was defined as right below the discomfort level or $\pm 20 \mathrm{~V}$, whichever lower amplitude. The medium amplitude was defined as an average between the low and high amplitudes. The three amplitudes of stimulation (low, medium, and high) were chosen to not produce direct motoric effects (muscle twitches).

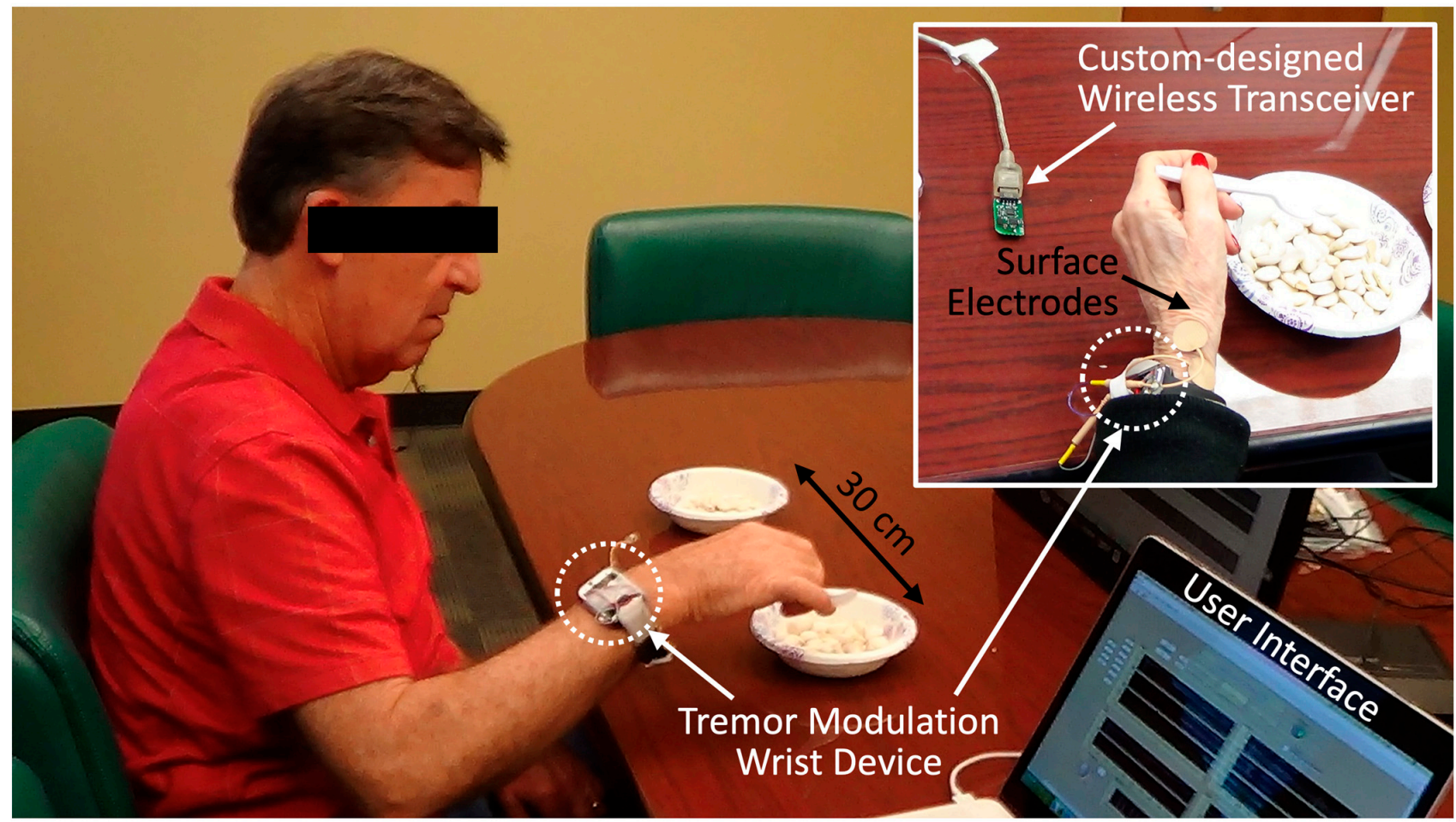

Figure 2. Experimental setup for peripheral nerve electrical stimulation using the wrist device while one of the participants performed the bean-transfer task. 

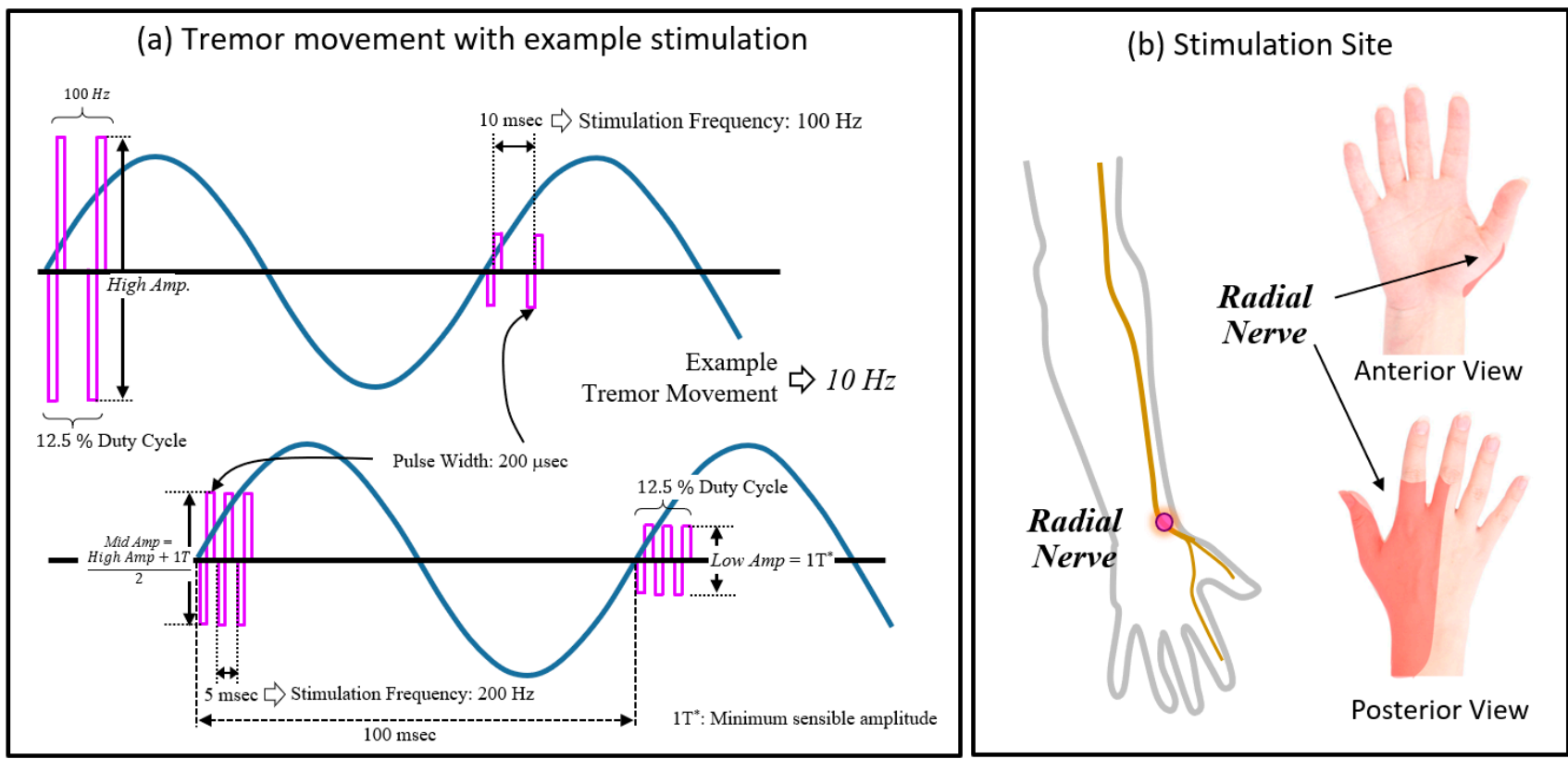

Figure 3. (a) Example electrical stimulation with tremor movement for various combinations of stimulation parameters; (b) stimulation site: radial nerves with their sensory nerve territories.

After completing the calibration and threshold setting, the participants took a fiveminute break to minimize the effects of stimulation on tremor movement. Thereafter, the computer generated various combinations of electrical stimulation parameters in random order, which were applied while the participants performed the bean-transfer task. Motion sensor data were continuously collected for use in the real-time tremor detection algorithm and for later evaluation of changes in tremor characteristics under the different stimulation settings. The total number of parameter combinations was 24 (three amplitude $\times$ two frequency $\times$ four phases) for each subject. Each combination of stimulation parameters was applied in one ten-second round, and there was a \pm 1.5 -min rest period between the rounds. We analyzed one bean-transfer as a trial; one round of stimulation consisted of four trials ( $\mathrm{n}=4$ for each stimulation setting).

\subsection{Performance Evaluation}

\subsubsection{Human Subjects}

This study was approved by the institutional review board (IRB) at Georgia Tech. We recruited nine ET participants between the ages of 47 and 82 (four males, median age 70). Written informed consent was obtained from each subject. A summary of the subject demographics is shown in Table 1. One of the subjects participated in two sessions, separated by three days. The subjects received their regular medication.

Table 1. Summary of subject demographics.

\begin{tabular}{cccc}
\hline Subject Demographics & $\mathbf{n ~ ( \% )}$ & Mean (SD) & Range \\
\hline Age & - & $67.67(11.67)$ & $47-82$ \\
Gender, male/female & $4(44 \%) / 5(56 \%)$ & - & \\
Years since diagnosis & - & $16.33(14.59)$ & $4-50$ \\
Device placement, right & $7(77.78 \%)$ & - & - \\
Medication & $5(56 \%)$ & - & - \\
TETRAS $(1-4)$ & - & $2.70(1.16)$ & $1-4$ \\
Dominant frequency $(\mathrm{Hz})$ & - & $5.86(1.26)$ & $3.60-9.20$ \\
Tremor power $\left(\mathrm{g}^{2} / \mathrm{Hz}\right)$ & - & $10.33(6.00)$ & $3.60-24.94$ \\
\hline
\end{tabular}


During an experimental session, we collected the subjects' tremor movements without stimulation (baseline tremor movement), and during periods of electrical stimulation of the radial nerve, administered at combinations of stimulation parameters (Table 2). To characterize baseline tremor, we also used the score from the Essential Tremor Rating Assessment Scale (TETRAS) [52,53]. TETRAS scores were based on maximal tremor amplitudes encountered while the participants performed the bean-transfer task (TETRAS $=0$ : no tremor; TETRAS $=1$ : barely visible $<1 \mathrm{~cm}$; TETRAS $=2: 1$ to $<5 \mathrm{~cm}$; TETRAS $=3: 5$ to $<20 \mathrm{~cm}$; and TETRAS $=4:>20 \mathrm{~cm}$ ).

Table 2. Stimulation parameters and their ranges.

\begin{tabular}{cc}
\hline Stimulation Parameters & Settings Used in This Study \\
\hline Amplitude & Low $=1 \mathrm{~T}$, Medium $=\frac{\text { Low }+ \text { Max }, \text { High }=\text { Max }}{2}$ \\
Pulse width & $200 \mu \mathrm{s}$ \\
Frequency & $100 \mathrm{~Hz}, 200 \mathrm{~Hz}$ \\
Duty cycle & $12.5 \%$ \\
Phase & Phase-locked to the tremor cycle at $0 \pi, \frac{1}{2} \pi, 1$ \\
$\mathrm{~T}=$ Sensory thresholds. & $\pi$, and $1 \frac{1}{2} \pi$ (Phase $1,2,3$, or 4, respectively). \\
\hline
\end{tabular}

\subsubsection{Tremor Output Metrics}

We high-pass filtered (HPF) the root-mean squared three-axis accelerometer data from the wrist device with a cutoff frequency of $3 \mathrm{~Hz}$ and extracted tremor frequency and tremor power. We selected tremor frequency as the peak frequency of the power spectral density (PSD) of the data during the bean-transfer task. We assigned an integral of the PSD between 4 and $12 \mathrm{~Hz}$ in the frequency domain as the tremor power (unit: $\mathrm{g}^{2} / \mathrm{Hz}$ ). The detailed analytical methods for the tremor output metrics appear in [47].

We defined the normalized tremor metrics as a ratio of the tremor output metrics of the control trial to those of the stimulation trial for each participant (see Equation (1)).

$$
\text { Normalized Tremor Metric }=\frac{\text { Tremor Output Metric of the Stimulation Trial }}{\text { Tremor Output Metric of the Control Trial }}
$$

If the ratio was close to 1 , it equaled a control movement, and if smaller than 1 , it indicated that stimulation had reduced tremor.

From a given trial with a certain stimulation parameter setting, we collected tremor movement for ten seconds and analyzed the data in four segments, each segment for one bean transfer. The average of the four segments was used to calculate the tremor metric.

\subsubsection{Stimulation Parameters and Combinations}

We used combinations of stimulation parameters, selected from three possible stimulation amplitudes, two stimulation frequencies, and phase-locked stimulation at one of four different phases of the tremor cycle. We selected combinations of stimulation parameters in random order. An example of the parameters and the combinations of parameters for the stimulation is shown in Figures $3 a$ and 4, and the stimulation parameters and their ranges are summarized in Table 2 . To determine the stimulation site, we placed a pair of electrodes over a branch of the radial nerve on the wrist and adjusted the placement of the electrodes so that the subjects sensed the stimulus in the desired location (see Figure $3 b$, highlighted area). 

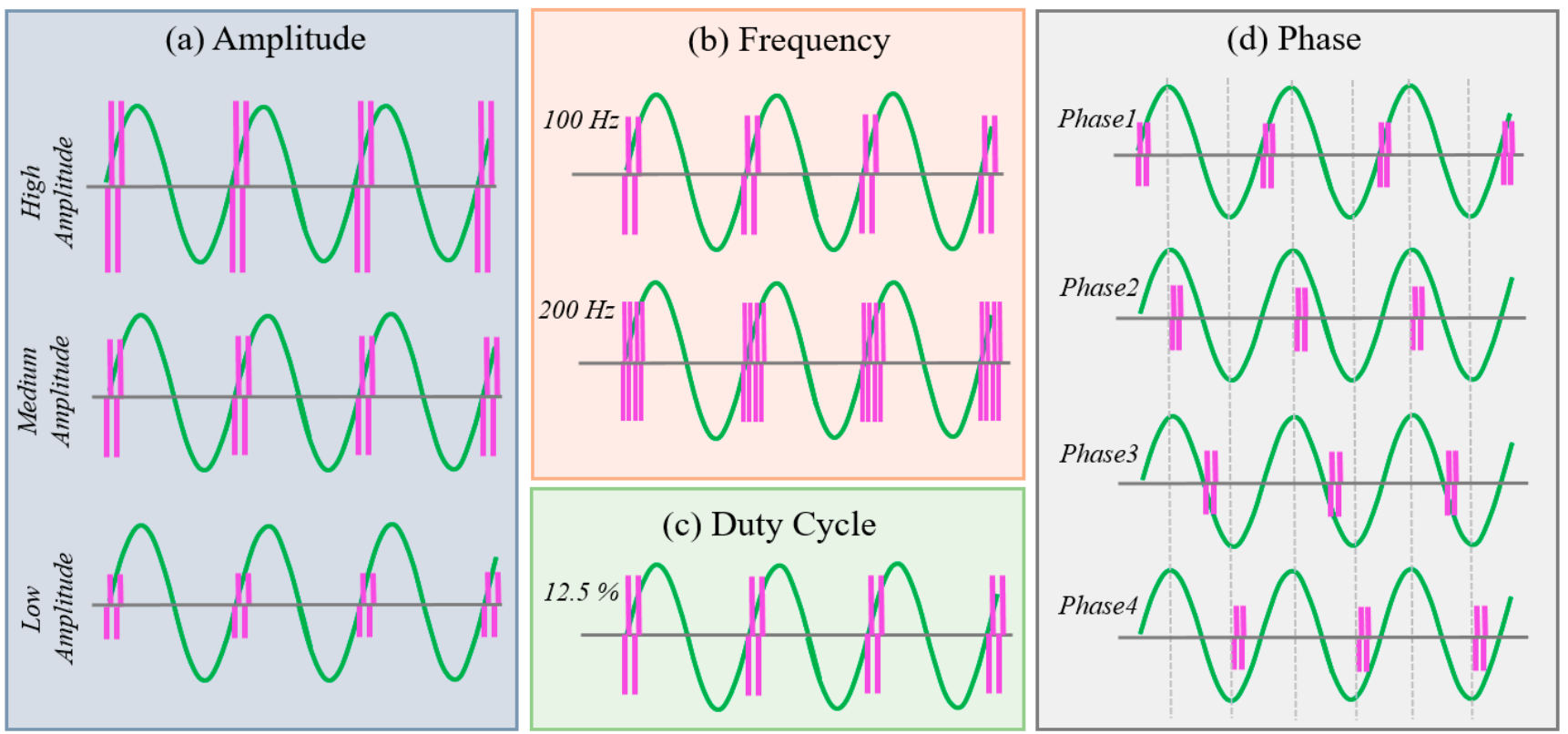

Figure 4. Stimulation parameters and their ranges: (a) amplitude, (b) frequency, (c) duty cycle, and (d) phase to the tremor cycle.

\subsection{Wearable Tremor Modulation System}

We developed a wearable tremor monitor and modulation system that includes custom-designed hardware and software (see Figure 5). We also implemented a tremor detection algorithm to be able to lock the stimulation to specific tremor phases.

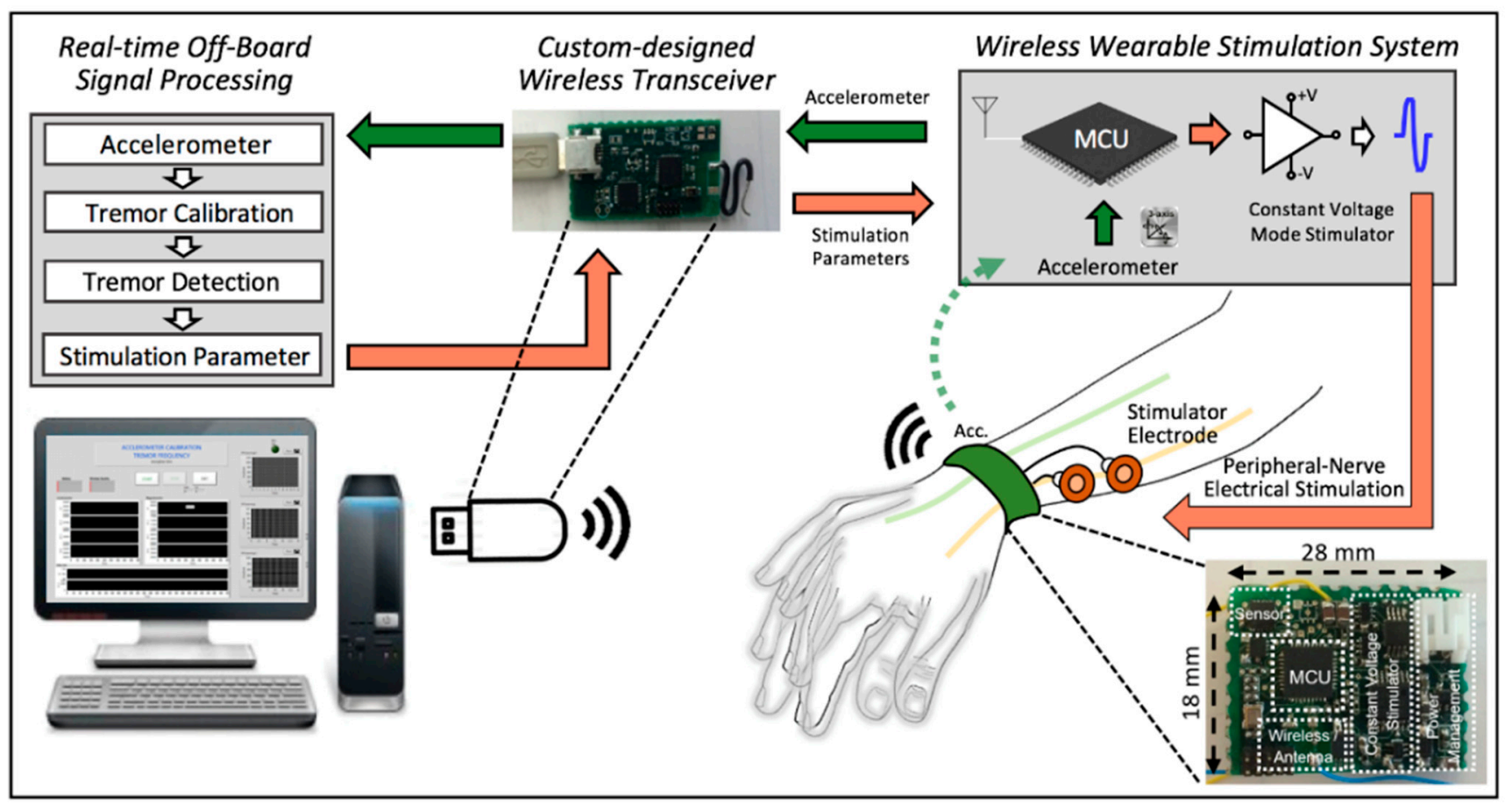

Figure 5. Overview of the real-time non-invasive tremor modulation system for essential tremor. 


\subsubsection{Tremor Modulation Hardware and Software}

The wearable wireless real-time closed-loop stimulation system collects/analyzes tremor movements and provides voltage-mode stimulation of peripheral nerves. The system consists of a $2.4 \mathrm{GHz}$ radio-frequency wireless transceiver and a pair of surface electrodes $\left(0.8^{\prime \prime}\right.$ round TENS Unit Electrodes, Syrtenty), and uses in its current iteration a computer with a graphical user interface (GUI) implemented on LabVIEW 2016 to help with tremor detection and modulation of parameters. In use, the electrodes are attached to the subject's skin over the targeted peripheral nerve (such as the radial nerve), allowing us to apply trains of bipolar stimulation to the nerve. Proper choice of stimulation parameters guarantees that the stimulation does not lead to (efferent) muscle stimulation but modulates afferent components of the nerve.

The wrist device contains a motion sensor (accelerometer, LSM303D, STMicroelectronics, Plan-les-Ouates, Switzerland) and custom-designed voltage mode stimulation circuitry, controlled by a microcontroller (CC2510, Texas Instruments, Texas, USA) with a built-in $2.4 \mathrm{GHz}$ RF transceiver. The electronics of the wrist device are mounted on an $18 \times 28 \mathrm{~mm}^{2}$ printed circuit board (PCB) with an enclosure that is fastened to a commercially available wrist band. The weight of the wrist device, including the battery and enclosure, is about $36 \mathrm{~g}$. The sampling rate of the motion sensor is $100 \mathrm{~Hz}$. The wrist device is powered with a rechargeable $3.7 \mathrm{~V}$ lithium-ion battery ( $300 \mathrm{mAh}$ ) and can run up to $20 \mathrm{~h}$ on a single charge. A full charge cycle (via a $5 \mathrm{~V}$ mini-USB connector) takes about three hours. The detailed hardware and software are described in more detail in [47].

Every $10 \mathrm{~ms}$, the wrist device conveys motion sensor data to a computer that runs the tremor detection algorithm, and receives, in turn, updated stimulation parameter information (stimulation amplitude, pulse width, frequency, duty cycle, phase, stimulation onset). Stimulation parameters are selected either manually or automatically. In the manual mode, the amplitude of the bi-phasic stimulus ( $\leq 20 \mathrm{~V} /$ phase) are applied to identify amplitude thresholds for each subject. The ranges of the parameters are summarized in Table 2. The threshold amplitudes of the minimum and maximum range had different settings. The three amplitudes of stimulation (low, medium, and high) were chosen to not produce direct motoric effects (muscle twitches).

\subsubsection{Tremor Detection Algorithm}

We first measured the dominant frequency and peak amplitude of the patient's tremor at baseline. We defined the "active tremor range" as tremor movements occurring at $>60 \%$ of the maximal tremor amplitude and within $\pm 30 \%$ of the dominant frequency. During the real-time "active tremor" detection mode for phase-locked stimulation, we utilized the high-pass filtered quadratic mean sensor output to apply this algorithm, and the tremor frequency was estimated by the sample differences from a five-sample moving window. Since the onset of tremor varies according to one's movements, the stimulation phase was not constant during the trial. For phase-locked stimulation (phases 1-4), we provided stimulation when the tremor was in both the active tremor range and at the selected phase.

\subsection{Statistical Analysis}

To analyze the overall effects of stimulation individually (Figure 6a,d), we conducted repeated measure one-way ANOVA test (ANOVA-1; $\alpha=0.05$ ). To analyze the effects of normalized tremor metrics by the TETRAS score group, we conducted two-way ANOVA tests (ANOVA-2; $\alpha=0.05$ ) for the normalized tremor metrics. We also conducted a pairwise comparison with the least significant difference (LSD) method of the repeated measure ANOVA test to analyze the difference between the normalized metrics. 
(a)

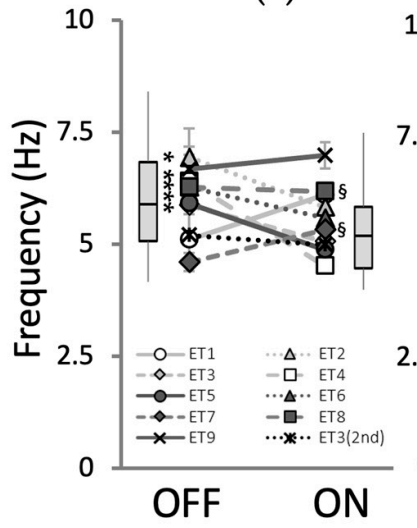

(d)

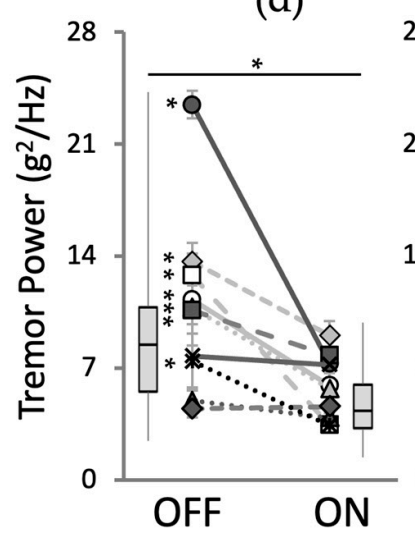

(b)

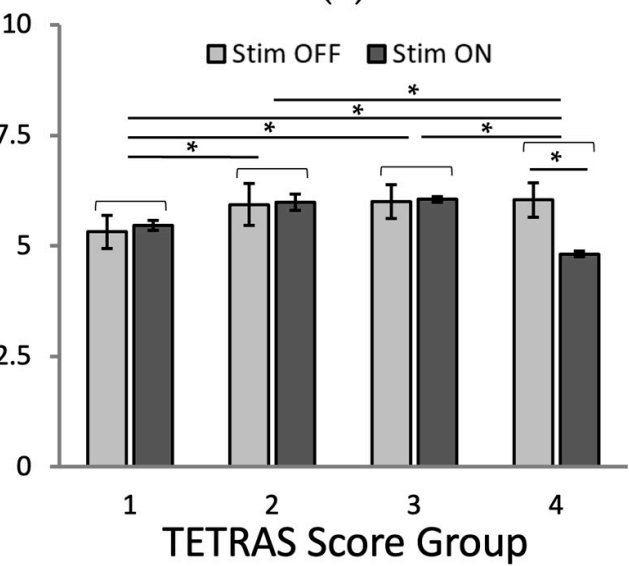

(e)

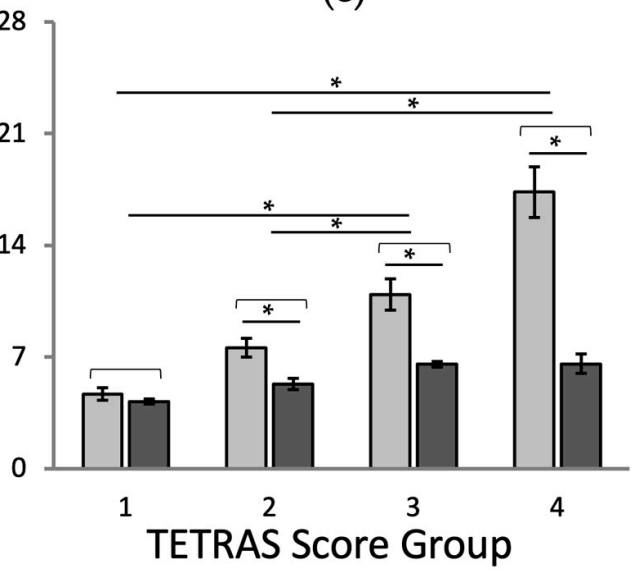

(c)

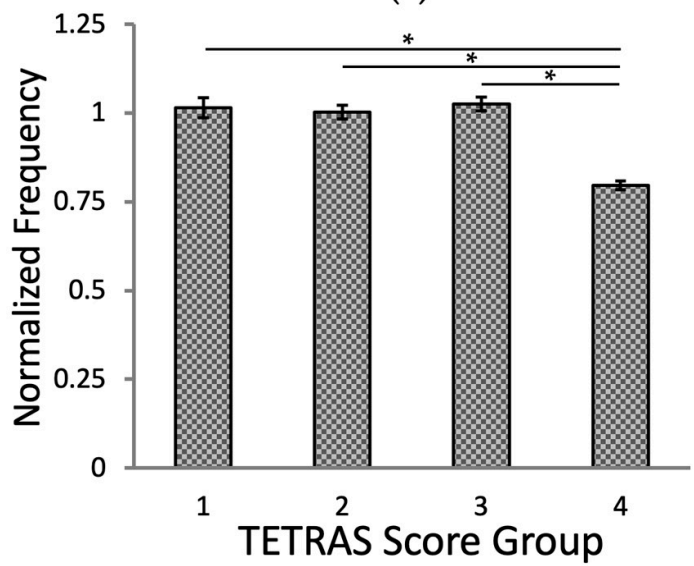

(f)

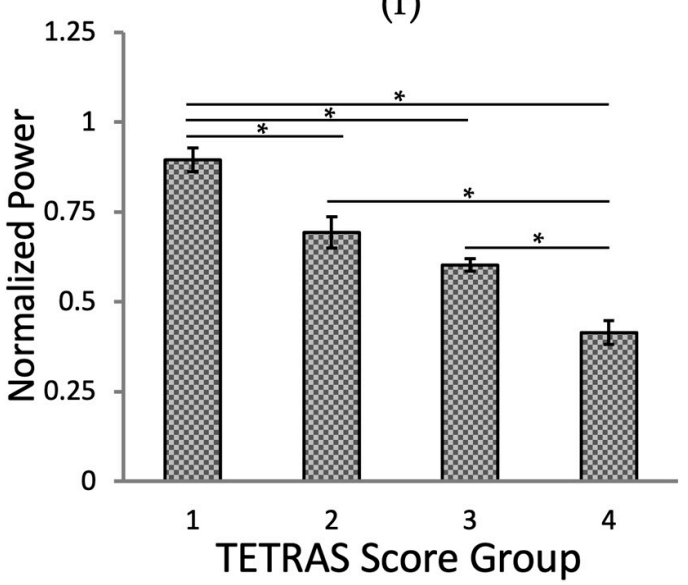

Figure 6. Effects of peripheral nerve stimulation. Overall tremor movement without and with stimulation for tremor metrics: dominant frequency (upper panels) and tremor power (bottom panels). The overall effect of stimulation (boxplots) with the effects of individual subjects for tremor metrics: (a) tremor frequency and (d) power. The effect of stimulation by TETRAS score groups for tremor metrics: (b) tremor frequency and (e) power. The normalized tremor metrics (metrics of stimulation/metrics of control) by TETRAS score group for tremor metrics: (c) tremor frequency and (f) power. The error bars represent standard errors of the mean. ${ }^{*}: p<0.05$ for significant decrease; $\S: p<0.05$ for significant increase.

\section{Results}

\subsection{Baseline Tremor Movements and General Effect of Stimulation}

As shown in Figure $6 \mathrm{a}, \mathrm{d}$, the stimulation (across all conditions) did not alter the dominant tremor frequency across all patients (no stimulation: $5.85 \pm 1.26 \mathrm{~Hz}$ vs. stimulation: $5.54 \pm 0.95 \mathrm{~Hz}$; ANOVA-1, $p=0.085$, followed by Fisher's LSD post hoc test), yet significantly reduced the tremor power (no stimulation: $10.93 \pm 6.00 \mathrm{~g}^{2} / \mathrm{Hz} \mathrm{vs}$. stim: $5.84 \pm 2.29 \mathrm{~g}^{2} / \mathrm{Hz}$; ANOVA-1, $p<0.001$, followed by Fisher's LSD post hoc test).

Analyzed in individual cases, there was a significant decrease in five patients (* in Figure $6 a$ ), no change in three patients, and a significant increase in two ( $\$$ in Figure $6 a$ ). Tremor power for all but one subject (ET7) decreased with stimulation; there was a significant tremor power reduction in seven patients (* in Figure $6 \mathrm{~d})$.

The analysis of the dominant frequency (Figure $6 \mathrm{~b}$; ANOVA-2, $p$ (stimulation) $=0.140$, $p($ TETRAS score group $)=0.004$, and $p$ (stimulation $\times x$ TETRAS score interaction $)=0.003$, followed by Fisher's LSD post hoc test) and power (Figure 6e; ANOVA-2, $p$ (stimulation, TETRAS score group, and stimulation $\times$ TETRAS score interaction) $<0.001$, followed by Fisher's LSD post hoc test) was significantly affected by the TETRAS score group. The 
normalized tremor metrics, grouped according to baseline TETRAS groups, also suggested that there was no significant tremor frequency change except for TETRAS $=4$ (Figure 6c); higher TETRAS score groups showed higher tremor power reduction ratio (Figure 6f).

\subsection{Effects of Stimulation Amplitude}

We did not see a significant reduction in tremor frequency between the no stimulation condition and the conditions using two different amplitudes of stimulation with all frequency and phases (Figure 7a; $p=0.134, p=0.086$, and $p=0.036$ for low, mid, and high, respectively), while tremor power was significantly reduced (Figure $7 \mathrm{c} ; p<0.001$ for all three amplitudes). Higher amplitudes of stimulation resulted in a greater reduction rate of tremor power (Figure 7c).

(a)

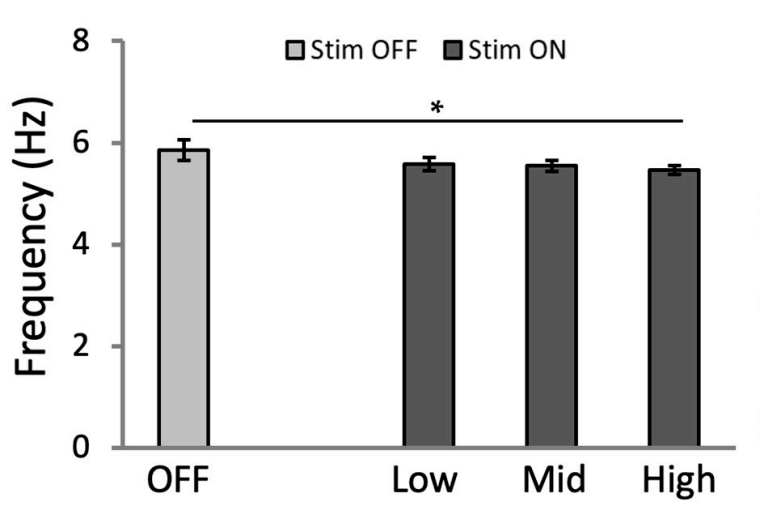

(c)

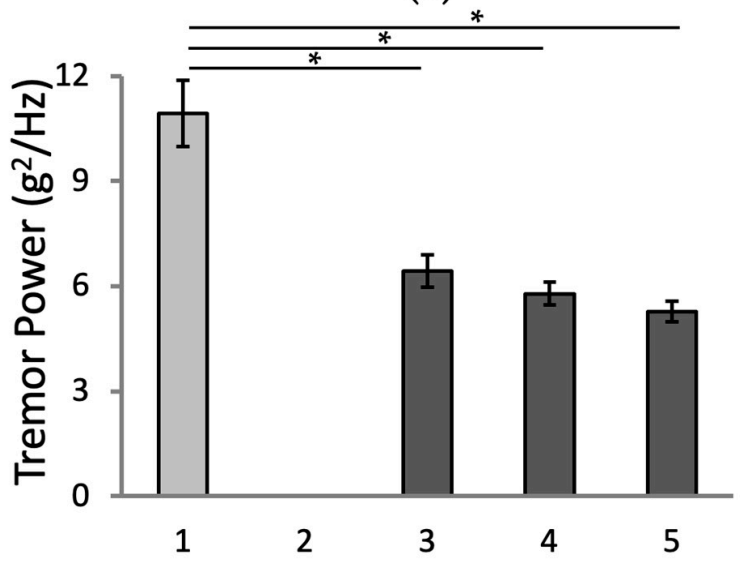

(b)

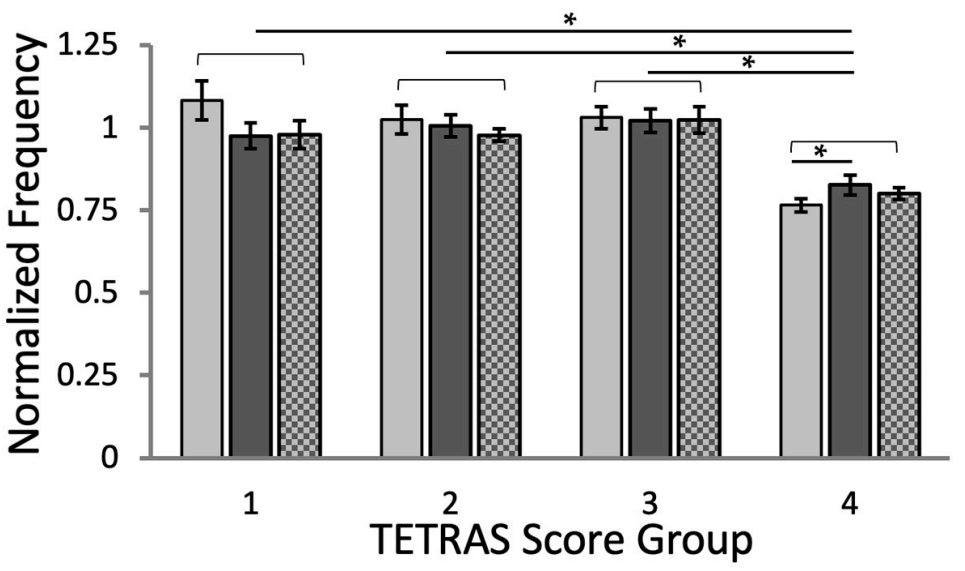

(d)

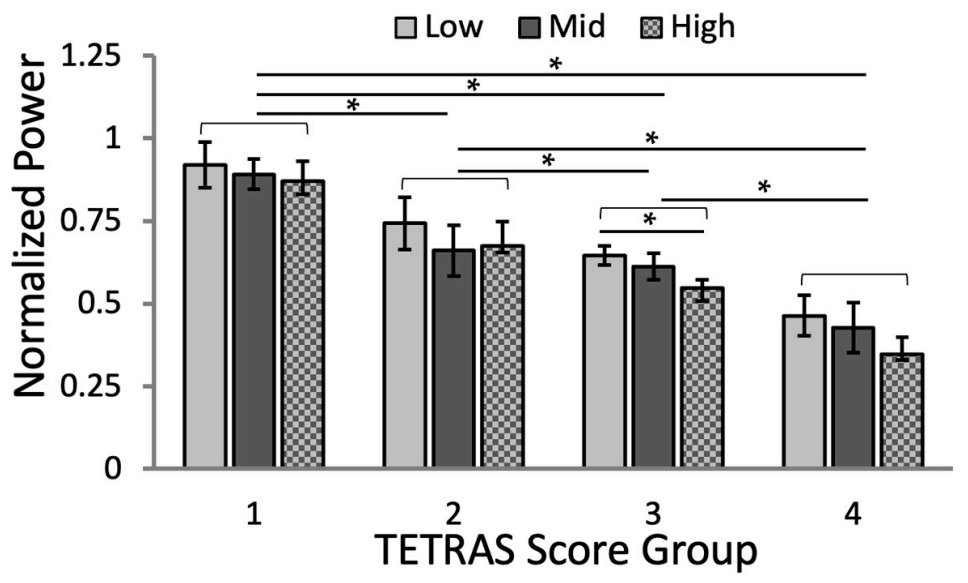

Figure 7. The effects of the stimulation amplitudes (low, mid, and high) by tremor metrics: (a) Tremor frequency and (c) power. The normalized tremor metrics for the different amplitudes by the TETRAS Score groups: (b) Tremor frequency and (d) power. The error bars represent the standard errors. $*: p<0.05$.

We carried out an analysis of the effects of stimulation amplitude and TETRAS score group for the normalized frequency and power values. The analysis of the normalized tremor frequency (Figure $7 \mathrm{~b}$; ANOVA-2, $p$ (amplitude) $=0.357, p$ (TETRAS score group) $<0.001$, and $p$ (amplitude $\times$ TETRAS score interaction $)=0.019$, followed by Fisher's LSD post hoc test $)$ and tremor power (Figure 7d; ANOVA-2, $p$ (amplitude, TETRAS score group, and amplitude $\times$ TETRAS score interaction) $<0.001$, followed by Fisher's LSD post hoc test), grouped according to pre-stimulation TETRAS scores, confirmed that there was a significant difference 
between the tremor amplitude and the TETRAS score interaction. There was no significant difference between the effects of different stimulation amplitudes when compared between TETRAS groups, except for TETRAS $=4$, where a significant difference between the low and mid amplitudes was found ( $p=0.046$; Figure $7 \mathrm{~b})$; the normalized tremor power, grouped according to TETRAS scores (Figure 7d) was not differentially influenced by stimulation amplitude, except for TETRAS $=3$ which showed a significant reduction at the highest stimulation amplitude $(p=0.027)$.

Based on these findings, we conclude that the stimulation does not appear to alter tremor frequency but has substantial effects on tremor power. It appears that the baseline tremor power is an important determinant of the overall success of stimulation.

\subsection{Effects of Stimulation Frequency}

We found no significant effect of the frequency of stimulation on tremor frequency (Figure 8a; $p=0.062$ and $p=0.064$ for 100 and $200 \mathrm{~Hz}$, respectively). Tremor power was significantly reduced by stimulation at either frequency (Figure 8c; $p<0.001$ for both frequencies), with no difference between 100 and $200 \mathrm{~Hz}$ stimulation.

(a)

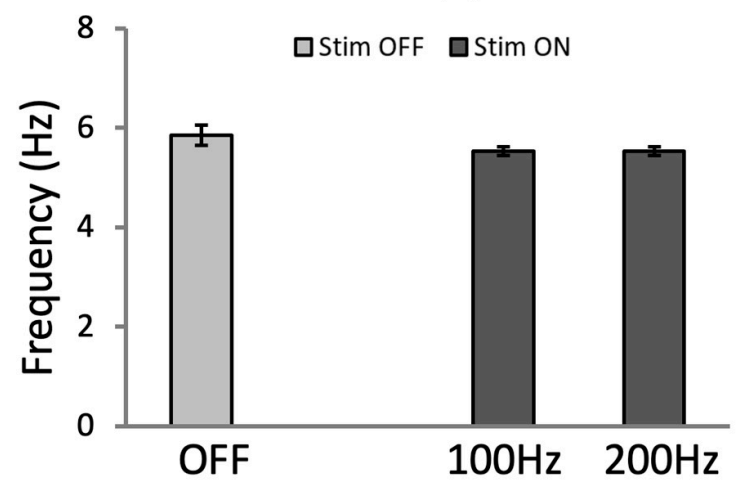

(c)

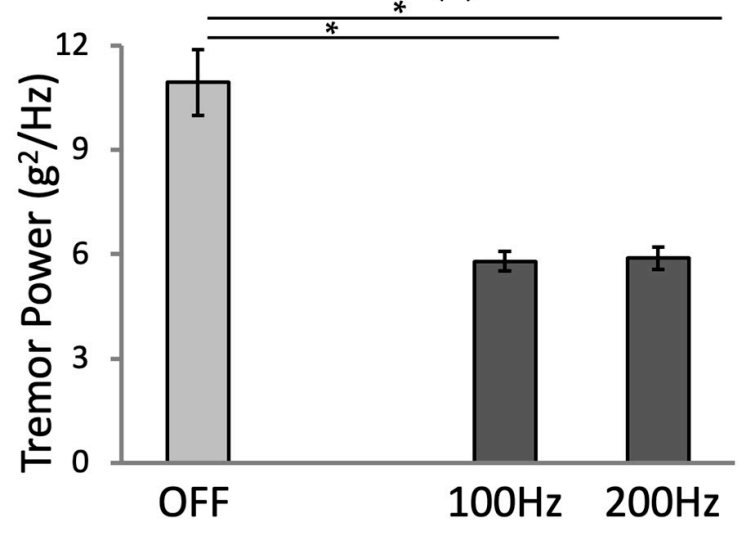

(b)

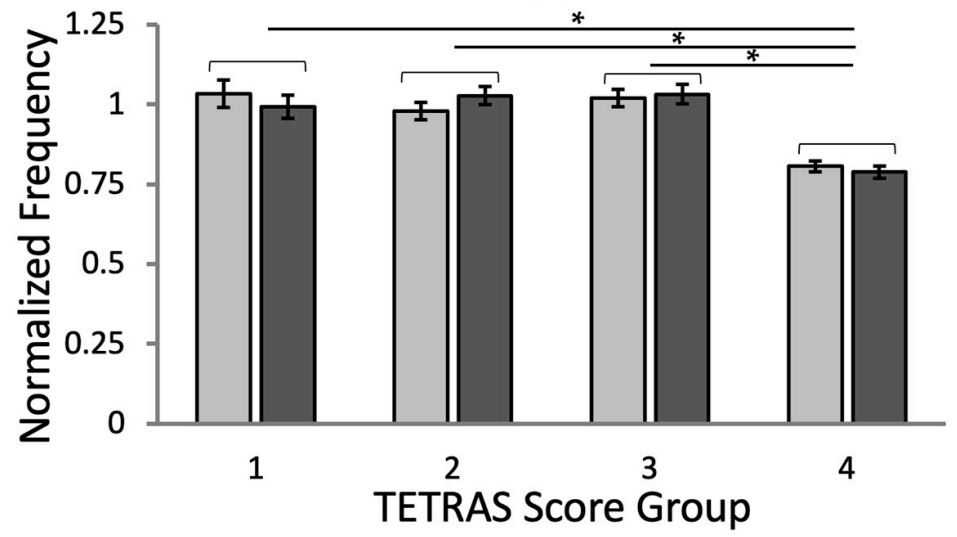

(d)

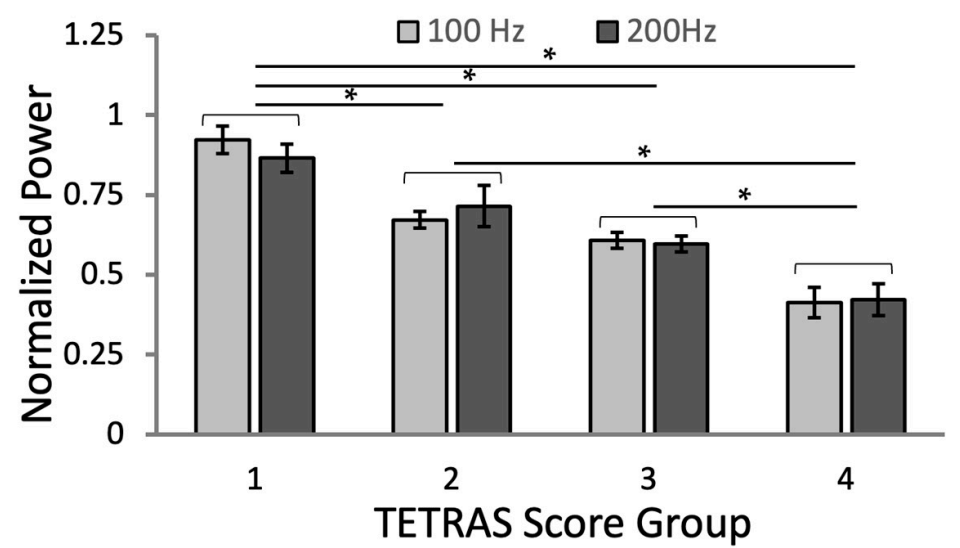

Figure 8. The effects of the stimulation frequency (100 and $200 \mathrm{~Hz}$ ) by tremor metrics: (a) Tremor frequency and (c) power. The normalized tremor metrics for the different frequency by the TETRAS score groups: (b) Tremor frequency and (d) power. The error bars represent the standard errors. $* p<0.05$.

We analyzed the effects of stimulation frequency and TETRAS score group for the normalized frequency and power values. The analysis of the normalized tremor frequency (Figure 8b; ANOVA-2, $p$ (frequency) $=0.991, p$ (TETRAS score group) $<0.001$, and $p$ (frequency $\times$ TETRAS score interaction) $=0.484$, followed by Fisher's LSD post hoc test) and tremor 
power (Figure 8d; ANOVA-2, $p$ (frequency) $=0.885, p$ (TETRAS score group) $<0.001$, and $p$ (frequency $\times$ TETRAS score interaction) $=0.809$, followed by Fisher's LSD post hoc test), grouped according to pre-stimulation TETRAS scores, confirmed that there was no significant difference between the stimulation frequencies and the interaction between frequencies and TETRAS scores.

\subsection{Effects of Stimulation Phases}

The tremor frequency was not altered by stimulation, regardless of the target tremor cycle phase, except for the phase at $1 \pi$ ( $p=0.014$; Figure 9a). However, the stimulation effects on tremor power differed between groups of data with stimulation in different tremor phases (Figure 9c; $p<0.001$ for all phases).

(a)

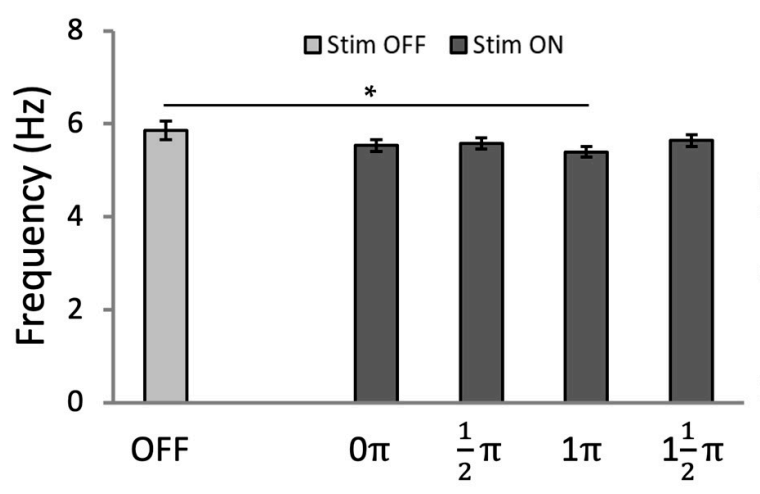

(c)

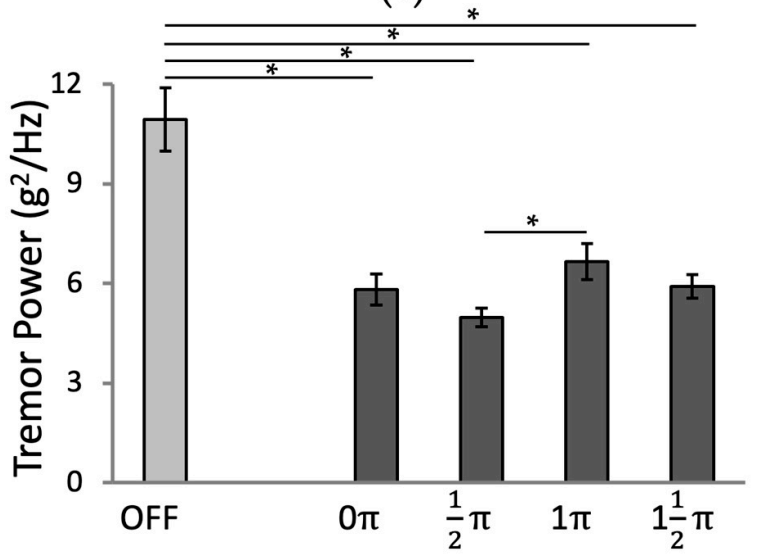

(b)

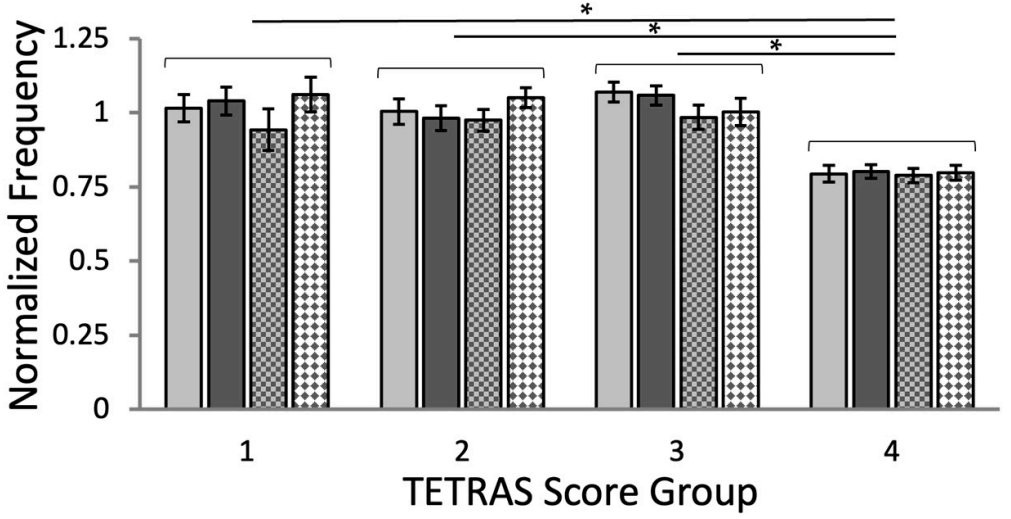

(d)

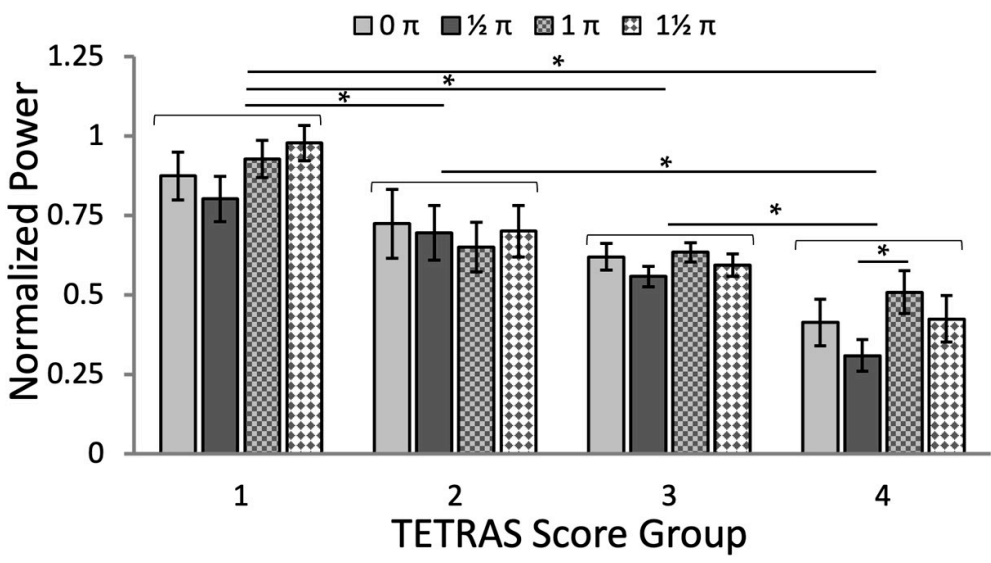

Figure 9. The effects of the phase-locked stimulation at four different phases by tremor metrics: (a) Tremor frequency and (c) power. The normalized tremor metrics for the different phases of stimulation by the TETRAS Score groups: (b) Tremor frequency and (d) power. The error bars represent the standard errors. ${ }^{*}: p<0.05$.

We also analyzed the effects of stimulation phase and TETRAS score group for the normalized frequency and power values. The analysis of the normalized tremor frequency (Figure 9b; ANOVA-2, $p$ (phase) $=0.182, p$ (TETRAS score group) $<0.001$, and $p$ (phase $\times$ TETRAS score interaction $)=0.103$, followed by Fisher's LSD post hoc test) and tremor power (Figure 9d; ANOVA-2, $p$ (phase, TETRAS score group, phase $\times$ TETRAS score interaction) $<0.001$, followed by Fisher's LSD post hoc test), grouped according to prestimulation TETRAS scores, confirmed that there were significant effects by the interaction between phases and TETRAS score groups for the normalized powers. From the pairwise comparison, the difference between $1 / 2 \pi$ and $1 \pi$ was significant $(p=0.039)$ for TETRAS $=4$, 
and the phase at $1 / 2 \pi$ showed higher tremor power reduction for TETRAS $=1,3$, and 4 (Figure $9 \mathrm{~d}$ ). The TETRAS $=3$ and 4 groups of records showed the most significant reduction in normalized power tremor with stimulation at phase $1 / 2 \pi$ (Figure $9 d$ ).

\section{Discussion}

Following up on our prior study of the effects of external stimulation on tremor [47], we explored the effect of stimulation amplitude, frequency, and tremor phase locking on tremor characteristics. We found that the tremor frequency remained unchanged by the stimulation, but that stimulation amplitude and phase influenced tremor power.

We found that the stimulation strongly affected tremor amplitude and frequency for the TETRAS = 4 group, and that tremor was more strongly reduced when the stimulation was applied out-of-phase in this group. The small sample size precludes meaningful analysis of this interesting finding. However, this should obviously be a topic of scientific and practical interest for a larger follow-up study.

Most published accounts of efforts to use sub-threshold electrical stimulation of afferent pathway to treat tremor [36-50] utilized a bulky (desktop) stimulation setup and showed tremor reductions of $14-60 \%$. A few other studies introduced wearable setups that applied electrical stimulation $[41,43,51]$. While these studies included a larger number of subjects, the effects of stimulation were only studied using constant stimulation parameters. Unlike the published studies, we adapted the stimulation to the tremor cycle in real-time and found significant benefits (up to $60 \%$ tremor reduction) with out-of-phase stimulation in TETRAS $=4$ patients. This finding supports the notion that tremor can be significantly reduced by out-of-phase sub-threshold stimulation [41,43], and suggests that the effects of stimulation can be optimized by adjusting stimulation parameters to ongoing tremor.

In this study, we evaluated the efficacy of the peripheral-nerve electrical stimulation with tremor metrics (dominant frequency and amplitude) using an accelerometer. However, stimulation-induced change in tremor metrics does not necessarily indicate a performance improvement for this task. This will be an important assessment in subsequent studies, aimed at investigating the practical usefulness of the device. Such assessments can be obtained using patient questionnaires, as done in our previous work [47]. Likewise, quantitative evaluations using a computer-based Fitts' law task [54-57] to evaluate the effects of stimulation on performance will be part of such studies.

This study was a small pilot experiment and had several limitations. It focused only on the effects of radial nerve stimulation. We may have noticed other effects of the stimulation parameters on tremor if we had applied stimulation to different sites (e.g., median and ulnar nerves). In addition, we analyzed results from only nine subjects. Even though we conducted multiple trials for each parameter combination ( $\mathrm{n}=24 \times 4$ for each participant), the number of sessions for each TETRAS group was only $2-3$. In future studies, it will be important to examine the effect of peripheral nerve stimulation on other sites and recruit more subjects and/or plan more sessions so that our conclusions are stronger. Larger group sizes may also allow additional subgroup analyses (for example, groups of different ages or genders).

Finally, even when acquired in the same patient, tremor measurements can substantially differ between sessions, perhaps depending on time of day, fatigue level, or medication states. While this variability limits the generalizability of the current study, it is also a strong argument in favor of continued development of a personalized and self-updating system to optimize stimulation effects on tremor in the future.

\section{Conclusions}

In this study, we investigated the effects of ranges of stimulation parameters in realtime phase-locked tremor modulation at the radial nerve. To evaluate the effect of the stimulation parameters, we collected motion sensor data of tremor movement with combinations of stimulation parameters compared to no stimulation and analyzed changes in dominant tremor frequency and tremor power. Combinations of the stimulation pa- 
rameters consisted of the following range of parameters: amplitude (low, medium, high), frequency $(100,200 \mathrm{~Hz}$ ), and phase (phase-locked stimulation at $0,1 / 2,1,11 / 2 \pi$ to tremor cycle). Although the stimulation frequency had a minor impact on tremor modulation, we concluded that (1) the stronger tremor group had a greater reduction in tremor power with a higher amplitude of stimulation, and (2) out-of-phase (at the \pm peak of tremor) phase-locked stimulation showed a greater reduction in tremor power in groups with more severe tremor. Particularly, we found that the effects of stimulation parameters on each subject depended on the current status of the tremor amplitude, so a real-time personalized optimization model for tremor modulation would help to increase the efficacy of tremor modulation. Despite the fact that the results of this study were based on a small number of participants and sessions, we strongly believe the findings have laid a solid foundation on which to optimize stimulation parameters, maximize the effects of tremor reduction, and minimize nerve fatigue and power consumption for consistent, long-term effects; ultimately, a promising alternative personalized treatment to existing Parkinson's Disease and related disorders treatment.

Author Contributions: Conceptualization, J.K., T.W., O.T.I. and S.P.D.; methodology, J.K. and S.P.D.; software, J.K.; validation, J.K.; formal analysis, J.K.; investigation, J.K., T.W., O.T.I. and S.P.D.; writingoriginal draft preparation, J.K., T.W., O.T.I. and S.P.D.; visualization, J.K.; supervision, T.W., O.T.I. and S.P.D.; project administration, J.K. and S.P.D. All authors have read and agreed to the published version of the manuscript.

Funding: This research was funded, in part, by NIH, Office of Research Infrastructure Programs, P51 OD011132 (T.W.) and NIH/NINDS grant P50 NS123103 (T.W.).

Institutional Review Board Statement: The study was conducted according to the guidelines of the Declaration of Helsinki and approved by the Institutional Review Board of the Georgia Institute of Technology (protocol code H15202 and date of approval: 20 September 2016).

Informed Consent Statement: Written informed consent has been obtained from the patient(s) to publish this paper.

Acknowledgments: The authors would like to thank the participants in our human-subject trials and the International Essential Tremor Foundation (IETF) Support Group in Savannah, Georgia, for their support in the recruitment of subjects for this study.

Conflicts of Interest: The authors declare no conflict of interest.

\section{References}

1. $\quad$ Bhatia, K.P.; Bain, P.; Bajaj, N.; Elble, R.J.; Hallett, M.; Louis, E.D.; Raethjen, J.; Stamelou, M.; Testa, C.M.; Deuschl, G. Tremor Task Force of the International Parkinson and Movement Disorder Society. Consensus Statement on the classification of tremors. from the task force on tremor of the International Parkinson and Movement Disorder Society. Mov. Disord. 2018, 33, 75-87. [CrossRef]

2. Shanker, V. Essential tremor: Diagnosis and management. BMJ 2019, 366, 14485. [CrossRef] [PubMed]

3. Hess, C.W.; Pullman, S.L. Tremor: Clinical phenomenology and assessment techniques. Tremor Other Hyperkinetic Mov. 2012, 2, 1-15. [CrossRef]

4. Bain, P.G. Tremor. Parkinsonism Relat. Disord. 2007, 13, S369-S374. [CrossRef]

5. Chandran, V.; Pal, P.K. Quality of life and its determinants in essential tremor. Parkinsonism Relat. Disord. 2013, 19, 62-65. [CrossRef]

6. Nguyen, H.V.; Ngian, V.; Cordato, D.; Shen, Q.; Chan, D.K. Quality of life in a random sample of community dwelling older patients with essential tremor. Acta Neurol. Scand. 2007, 116, 289-292. [CrossRef]

7. Louis, E.D.; Machado, D.G. Tremor-related quality of life: A comparison of essential tremor vs. Parkinson's disease patients. Parkinsonism Relat. Disord. 2015, 21, 729-735. [CrossRef] [PubMed]

8. Sullivan, K.L.; Hauser, R.A.; Zesiewicz, T.A. Essential tremor: Epidemiology, diagnosis, and treatment. Neurology 2004, 10, 250-258. [CrossRef]

9. 12-Month Annual Report, Hope through Research, Awareness and Support. Available online: http:/ /www.essentialtremor.org/ wp-content/uploads / 2013/07/2006-IETF-An-Rep-for-WEB.pdf (accessed on 22 September 2021).

10. Thenganatt, M.A.; Jankovic, J. The relationship between essential tremor and Parkinson's disease. Parkinsonism Relat. Disord. 2016, 22, S162-S165. [CrossRef]

11. Baumann, C.R. Epidemiology, diagnosis and differential diagnosis in Parkinson's disease tremor. Parkinsonism Relat. Disord. 2012, 18, S90-S92. [CrossRef] 
12. Bhidayasiri, R. Differential diagnosis of common tremor syndromes. Postgrad. Med. J. 2005, 81, 756-762. [CrossRef]

13. De Lau, L.M.; Breteler, M.M. Epidemiology of Parkinson's disease. Lancet Neurol. 2006, 5, 525-535. [CrossRef]

14. Louis, E.D.; Ferreira, J.J. How common is the most common adult movement disorder? Update on the worldwide prevalence of essential tremor. Mov. Disord. 2010, 25, 534-541. [CrossRef] [PubMed]

15. Mayo Clinic. Essential Tremor: Treatment. Available online: http://www.mayoclinic.org/diseases-conditions/essential-tremor/ basics / treatment/con-20034509 (accessed on 22 September 2021).

16. Drug Treatments for Essential Tremor. Available online: https://www.webmd.com/epilepsy/guide/essential-tremor-drug (accessed on 22 September 2021).

17. Ferreira, J.J.; Mestre, T.A.; Lyons, K.E.; Benito-León, J.; Tan, E.K.; Abbruzzese, G.; Hallett, M.; Haubenberger, D.; Elble, R.; Deuschl, G. evidence-based review of treatments for essential tremor. Mov. Disord. 2019, 34, 950-958. [CrossRef]

18. Louis, E.D.; Rios, E.; Henchcliffe, C. How are we doing with the treatment of essential tremor (ET)? Persistence of patients with ET on medication: Data from 528 patients in three settings. Eur. J. Neurol. 2010, 17, 882-884. [CrossRef] [PubMed]

19. Louis, E.D. The roles of age and aging in essential tremor: An epidemiological perspective. Neuroepidemiology 2019, 52, 111-118. [CrossRef] [PubMed]

20. Jankovic, J.; Schwartz, K. Botulinum toxin treatment of tremors. Neurology 1991, 41, 1185. [CrossRef]

21. Pahwa, R.; Busenbark, K.; Swanson-Hyland, E.F.; Dubinsky, R.M.; Hubble, J.P.; Gray, C.; Koller, W.C. Botulinum toxin treatment of essential head tremor. Neurology 1995, 45, 822-824. [CrossRef]

22. Warrick, P.; Dromey, C.; Irish, J.C.; Durkin, L.; Pakiam, A.; Lang, A. Botulinum toxin for essential tremor of the voice with multiple anatomical sites of tremor: A crossover design study of unilateral versus bilateral injection. Laryngoscope 2000, 110, 1366-1374. [CrossRef] [PubMed]

23. Kumar, R.; Lozano, A.M.; Kim, Y.J.; Hutchison, W.D.; Sime, E.; Halket, E.; Lang, A.E. Double-blind evaluation of subthalamic nucleus deep brain stimulation in advanced Parkinson's disease. Neurology 1998, 51, 850-855. [CrossRef] [PubMed]

24. Hubble, J.P.; Busenbark, K.L.; Wilkinson, S.; Penn, R.D.; Lyons, K.; Koller, W.C. Deep brain stimulation for essential tremor. Neurology 1996, 46, 1150-1153. [CrossRef]

25. Lyons, K.E.; Pahwa, R. Deep brain stimulation and tremor. Neurotherapeutics 2008, 5, 331-338. [CrossRef]

26. Fasano, A.; Lozano, A.M.; Cubo, E. New neurosurgical approaches for tremor and Parkinson's disease. Curr. Opin. Neurol. 2017, 30, 435-446. [CrossRef]

27. Dallapiazza, R.F.; Lee, D.J.; De Vloo, P.; Fomenko, A.; Hamani, C.; Hodaie, M.; Kalia, S.K.; Fasano, A.; Lozano, A.M. Outcomes from stereotactic surgery for essential tremor. J. Neurol. Neurosurg. Psychiatry 2019, 90, 474-482. [CrossRef]

28. Al-Fatly, B.; Ewert, S.; Kübler, D.; Kroneberg, D.; Horn, A.; Kühn, A.A. Connectivity profile of thalamic deep brain stimulation to effectively treat essential tremor. Brain 2019, 142, 3086-3098. [CrossRef] [PubMed]

29. Cury, R.G.; Fraix, V.; Castrioto, A.; Fernández, M.A.; Krack, P.; Chabardes, S.; Seigneuret, E.; Alho, E.J.; Benabid, A.L.; Moro, E. Thalamic deep brain stimulation for tremor in Parkinson disease, essential tremor, and dystonia. Neurology 2017, 89, 1416-1423. [CrossRef] [PubMed]

30. Prochazka, A.; Elek, J.; Javidan, M. Attenuation of pathological tremors by functional electrical stimulation I: Method. Ann. Biomed. Eng. 1992, 20, 205-224. [CrossRef] [PubMed]

31. Javidan, M.; Elek, J.; Prochazka, A. Attenuation of pathological tremors by functional electrical stimulation II: Clinical evaluation. Ann. Biomed. Eng. 1992, 20, 225-236. [CrossRef] [PubMed]

32. Gillard, D.M.; Cameron, T.; Prochazka, A.; Gauthier, M.J. Tremor suppression using functional electrical stimulation: A comparison between digital and analog controllers. IEEE Trans. Rehabil. Eng. 1999, 7, 385-388. [CrossRef] [PubMed]

33. Bó, A.P.L.; Azevedo-Coste, C.; Geny, C.; Poignet, P.; Fattal, C. On the use of fxedintensity functional electrical stimulation for attenuating essential tremor. Artif Organs. 2014, 38, 984-991. [CrossRef]

34. Gallego, J.A.; Rocon, E.; Manuel Belda-Lois, J.; Pons, J.L. A neuroprosthesis for tremor management through the control of muscle co-contraction. J. Neuroeng. Rehabil. 2013, 10, 36. [CrossRef]

35. Grimaldi, G.; Camut, S.; Manto, M. Functional electrical stimulation efect on upper limb tremor. Int. J. Bioelectromagn. 2011, 13, 123-124.

36. Jitkritsadakul, O.; Thanawattano, C.; Anan, C.; Bhidayasiri, R. Exploring the effect of electrical muscle stimulation as a novel treatment of intractable tremor in Parkinson's disease. J. Neurol. Sci. 2015, 358, 146-152. [CrossRef] [PubMed]

37. Hao, M.; He, X.; Xiao, Q.; Alstermark, B.; Lan, N. Corticomuscular transmission of tremor signals by propriospinal neurons in Parkinson's disease. PLoS ONE 2013, 8, e79829. [CrossRef] [PubMed]

38. Heo, J.H.; Kim, J.W.; Kwon, Y.; Lee, S.K.; Eom, G.M.; Kwon, D.Y.; Lee, C.N.; Park, K.W.; Manto, M. Sensory electrical stimulation for suppression of postural tremor in patients with essential tremor. Bio-Med. Mater. Eng. 2015, 26, S803-S809. [CrossRef] [PubMed]

39. Heo, J.H.; Jeon, H.M.; Choi, E.B.; Kwon, D.Y.; Eom, G.M. Continuous sensory electrical stimulation for the suppression of parkinsonian rest tremor. J. Mech. Med. Biol. 2018, 18, 1840006. [CrossRef]

40. Heo, J.H.; Jeon, H.M.; Choi, E.B.; Kwon, D.Y.; Eom, G.M. Effect of Sensory Electrical Stimulation on Resting Tremors in Patients with Parkinson's Disease and SWEDDS. J. Mech. Med. Biol. 2019, 19, 1940033. [CrossRef] 
41. Pahwa, R.; Dhall, R.; Ostrem, J.; Gwinn, R.; Lyons, K.; Ro, S.; Dietiker, C.; Luthra, N.; Chidester, P.; Hamner, S.; et al. An acute randomized controlled trial of noninvasive peripheral nerve stimulation in essential tremor. Neuromodulation Technol. Neural Interface 2019, 22, 537-545. [CrossRef]

42. Dideriksen, J.L.; Laine, C.M.; Dosen, S.; Muceli, S.; Rocon, E.; Pons, J.L.; Benito-Leon, J.; Farina, D. Electrical stimulation of afferent pathways for the suppression of pathological tremor. Front. Neurosci. 2017, 11, 178. [CrossRef]

43. Isaacson, S.H.; Peckham, E.; Tse, W.; Waln, O.; Way, C.; Petrossian, M.T.; Dahodwala, N.; Soileau, M.J.; Lew, M.; Dietiker, C.; et al. Prospective home-use study on non-invasive neuromodulation therapy for essential tremor. Tremor Other Hyperkinetic Mov. 2020, 10, 29. [CrossRef]

44. $\quad$ Lin, P.T.; Ross, E.K.; Chidester, P.; Rosenbluth, K.H.; Hamner, S.R.; Wong, S.H.; Sanger, T.D.; Hallett, M.; Delp, S.L. Noninvasive neuromodulation in essential tremor demonstrates relief in a sham-controlled pilot trial. Mov. Disord. 2018, 33, 1182. [CrossRef]

45. Pascual-Valdunciel, A.; González-Sánchez, M.; Muceli, S.; Adán-Barrientos, B.; Escobar-Segura, V.; Pérez-Sánchez, J.R.; Jung, M.K.; Schneider, A.; Hoffmann, K.P.; Moreno, J.C.; et al. Intramuscular stimulation of muscle afferents attains prolonged tremor reduction in essential tremor patients. IEEE Trans. Biomed. Eng. 2020, 68, 1768-1776. [CrossRef]

46. Reis, C.; Arruda, B.S.; Pogosyan, A.; Brown, P.; Cagnan, H. Essential tremor amplitude modulation by median nerve stimulation. Sci. Rep. 2021, 11, 17720. [CrossRef] [PubMed]

47. Kim, J.; Wichmann, T.; Inan, O.T.; Deweerth, S.P. A wearable system for attenuating essential tremor based on peripheral nerve stimulation. IEEE J. Transl. Eng. Health Med. 2020, 8, 1-11. [CrossRef]

48. Karamesinis, A.; Sillitoe, R.V.; Kouzani, A.Z. Wearable Peripheral Electrical Stimulation Devices for the Reduction of Essential Tremor: A Review. IEEE Access 2021, 9, 80066-80076. [CrossRef]

49. Pascual-Valdunciel, A.; Hoo, G.W.; Avrillon, S.; Barroso, F.O.; Goldman, J.G.; Hernandez-Pavon, J.C.; Pons, J.L. Peripheral electrical stimulation to reduce pathological tremor: A review. J. Neuroeng. Rehabil. 2021, 18, 33. [CrossRef]

50. Lora-Millan, J.S.; Delgado-Oleas, G.; Benito-León, J.; Rocon, E. A Review on Wearable Technologies for Tremor Suppression. Front. Neurol. 2021, 12, 700600. [CrossRef] [PubMed]

51. CalaTM Health Care Professional FAQs. Available online: https://calatrio.com/healthcare-professionals/frequently-askedquestions/ (accessed on 22 September 2021)

52. Elble, R.J.; Pullman, S.L.; Matsumoto, J.Y.; Raethjen, J.; Deuschl, G.; Tintner, R. Tremor amplitude is logarithmically related to 4-and 5-point tremor rating scales. Brain 2006, 129, 2660-2666. [CrossRef]

53. Elble, R.J. The essential tremor rating assessment scale. J. Neurol. Neuromedicine 2016, 1, 34-38.

54. Kim, J.; Wichmann, T.; Inan, O.T.; Deweerth, S.P. Fitts Law-Based Performance Metrics to Quantify Tremor in Individuals with Essential Tremor. IEEE J. Biomed. Health Inform. 2021, 1-11. [CrossRef]

55. Kim, J. Assessment and modulation of essential tremor using peripheral-nerve stimulation. Ph.D. Thesis, Georgia Institute of Technology, Atlanta, GA, USA, 1 January 2018.

56. Kim, J.; Parnell, C.; Wichmann, T.; Deweerth, S.P. Longitudinal wearable tremor measurement system with activity recognition algorithms for upper limb tremor. In Proceedings of the 2016 38th Annual International Conference of the IEEE Engineering in Medicine and Biology Society (EMBC), Orlando, FL, USA, 16-20 August 2016; pp. 6166-6169.

57. Kim, J.; Parnell, C.; Wichmann, T.; Deweerth, S.P. Quantitative assessment of arm tremor in people with neurological disorders. In Proceedings of the 2016 38th Annual International Conference of the IEEE Engineering in Medicine and Biology Society (EMBC), Orlando, FL, USA, 16-20 August 2016; pp. 2299-2302. 